\title{
Morozov's Discrepancy Principle under General Source Conditions
}

\author{
M. T. Nair, E. Schock and U. Tautenhahn
}

\begin{abstract}
In this paper we study linear ill-posed problems $A x=y$ in a Hilbert space setting where instead of exact data $y$ noisy data $y^{\delta}$ are given satisfying $\left\|y-y^{\delta}\right\| \leq \delta$ with known noise level $\delta$. Regularized approximations are obtained by a general regularization scheme where the regularization parameter is chosen from Morozov's discrepancy principle. Assuming the unknown solution belongs to some general source set $M$ we prove that the regularized approximation provides order optimal error bounds on the set $M$. Our results cover the special case of finitely smoothing operators $A$ and extend recent results for infinitely smoothing operators.
\end{abstract}

Keywords: Linear ill-posed problems, regularization, discrepancy principle, general source conditions, order optimal error bounds

AMS subject classification: $65 \mathrm{~J} 20$

\section{Introduction}

Ill-posed problems arise in several contexts and have important applications in science and engineering (see, e.g., $[1,3,4,6,10,13])$. In this paper we are interested in the minimum-norm solution $x^{\dagger} \in X$ of the ill-posed problem

$$
A x=y
$$

where $A: X \rightarrow Y$ is a linear bounded operator with non-closed range $R(A)$ of $A, y \in R(A)$ and $X, Y$ are infinite dimensional real Hilbert spaces with

M. Thamban Nair: Dept. Math. of the Univ., P.O. Box 3049, D-67653 Kaiserslautern; Home Institution: Indian Inst. Techn. Madras, Dept. Math., Chennai 600 036, India

E. Schock: Dept. Math. of the Univ., P.O. Box 3049, D-67653 Kaiserslautern

U. Tautenhahn: Dept. Math. of the Univ. Appl. Sci. Zittau/Görlitz, P.O. Box 1454, D-02754 Zittau

mtnair@iitm.ac.in, schock@mathematik.uni-kl.de, u.tautenhahn@hs-zigr.de

ISSN 0232-2064 / \$2.50 C Heldermann Verlag Berlin 
inner products $(\cdot, \cdot)$ and norms $\|\cdot\|$, respectively. Throughout this paper we assume that $y^{\delta} \in Y$ are the available noisy data with $\left\|y-y^{\delta}\right\| \leq \delta$ and known noise level $\delta>0$. For the stable approximate solution of problem (1.1) some regularization technique has to be applied, which provides regularized approximations $x_{\alpha}^{\delta}=R_{\alpha}^{\delta} y^{\delta}$ with property $x_{\alpha}^{\delta} \rightarrow x^{\dagger}$ as $\delta \rightarrow 0$ where the regularization parameter $\alpha=\alpha\left(\delta, y^{\delta}\right)$ has to be chosen properly. Hence, regularized approximations $x_{\alpha}^{\delta}$ depend continuously on the data. However, the convergence of $x_{\alpha}^{\delta}$ to $x^{\dagger}$ can be arbitrarily slow without assuming additional quantitative a priori restrictions on the unknown solution $x^{\dagger}$, which is typical for ill-posed problems (see [12]). In order to guarantee certain convergence rates the set of solutions has to be restricted to certain source sets. Typically, for operator equations (1.1) with finitely smoothing operators $A$, source conditions of the type $x^{\dagger} \in M_{p, E}$ with

$$
M_{p, E}=\left\{x \in X \mid x=\left(A^{*} A\right)^{\frac{p}{2}} v,\|v\| \leq E\right\} \quad(p>0)
$$

are exploited (see $[1,2,6,15])$. For infinitely smoothing operators $A$ source conditions of type (1.2) are generally too restrictive. In this case it is natural to assume that $x^{\dagger} \in M_{p, E}^{\log }$ with

$$
M_{p, E}^{\log }=\left\{x \in X \mid x=\ln ^{-\frac{p}{2}}\left(A^{*} A\right)^{-1} v,\|v\| \leq E\right\} \quad(p>0)
$$

(see $[5,7,11,13])$. In this paper we are interested in order optimality results under general source conditions $x^{\dagger} \in M_{\varphi, E}$ with $M_{\varphi, E}$ given by

$$
M_{\varphi, E}=\left\{x \in X \mid x=\left[\varphi\left(A^{*} A\right)\right]^{\frac{1}{2}} v,\|v\| \leq E\right\} .
$$

Herein the operator function $\varphi\left(A^{*} A\right)$ is well defined via spectral representation $\varphi\left(A^{*} A\right)=\int_{0}^{a} \varphi(\lambda) d E_{\lambda}$ where $A^{*} A=\int_{0}^{a} \lambda d E_{\lambda}$ is the spectral representation of $A^{*} A,\left\{E_{\lambda}: 0 \leq \lambda \leq a\right\}$ is the spectral family of $A^{*} A$ with $a>0$ satisfying $\sigma\left(A^{*} A\right) \subseteq[0, a]$ and $\|A\|^{2} \leq a, \sigma\left(A^{*} A\right)$ denoting the spectrum of the operator $A^{*} A$. Throughout this paper we assume that $x^{\dagger} \in M_{\varphi, E}$ such that

$$
x^{\dagger}=\left[\varphi\left(A^{*} A\right)\right]^{\frac{1}{2}} v \quad \text { with } \quad\|v\| \leq E
$$

where the function $\varphi$ satisfies

Assumption 1.1. The function $\varphi:(0, a] \rightarrow(0, \infty)$ with $\left\|A^{*} A\right\| \leq a$ is continuous and satisfies the conditions

(i) $\lim _{\lambda \rightarrow 0} \varphi(\lambda)=0$

(ii) $\varphi$ is strictly monotonically increasing on $(0, a]$

(iii) $\rho:(0, \varphi(a)] \rightarrow(0, a \varphi(a)]$ defined by $\rho(\lambda)=\lambda \varphi^{-1}(\lambda)$ is convex. 


\section{Optimality and order optimality}

Any operator $R: Y \rightarrow X$ can be considered as a special method for solving equation (1.1). The approximate solution to (1.1) is then given by $R y^{\delta}$. Let us consider the worst case error $\Delta(\delta, R)$ for identifying the minimum-norm solution $x^{\dagger}$ of problem (1.1) from $y^{\delta} \in Y$ under the assumptions $\left\|y-y^{\delta}\right\| \leq \delta$ and $x^{\dagger} \in M_{\varphi, E}$ defined by

$$
\Delta(\delta, R)=\sup \left\{\left\|R y^{\delta}-x^{\dagger}\right\|: x^{\dagger} \in M_{\varphi, E}, y^{\delta} \in Y,\left\|y-y^{\delta}\right\| \leq \delta\right\}
$$

This worst case error characterizes the maximal error of the method $R$ if the minimum-norm solution $x^{\dagger}$ of problem (1.1) varies in the set $M_{\varphi, E}$. An optimal method $R_{0}$ is characterized by

$$
\Delta\left(\delta, R_{0}\right)=\inf _{R} \Delta(\delta, R)
$$

It is easy to see that

$$
\inf _{R} \Delta(\delta, R) \geq \omega\left(\delta, M_{\varphi, E}\right)
$$

where

$$
\omega\left(\delta, M_{\varphi, E}\right)=\sup \left\{\|x\|: x \in M_{\varphi, E},\|A x\| \leq \delta\right\} .
$$

In view of the above relation, it is important to have some estimates for the quantity $\omega\left(\delta, M_{\varphi, E}\right)$. From [13] we have

Theorem 2.1. Let $M_{\varphi, E}$ be given by (1.4) and let Assumption 1.1 be satisfied. Then

$$
\omega\left(\delta, M_{\varphi, E}\right) \leq E \sqrt{\rho^{-1}\left(\frac{\delta^{2}}{E^{2}}\right)} .
$$

If $\frac{\delta^{2}}{E^{2}} \in \sigma\left(A^{*} A \varphi\left(A^{*} A\right)\right)$, then in (2.2) equality holds.

Due to Theorem 2.1, the following definition makes sense.

Definition 2.2. Consider the set $M_{\varphi, E}$ of (1.4) and let Assumption 1.1 be satisfied. Then any regularization method $R=R_{\alpha}^{\delta}$ or any regularized approximation $x_{\alpha}^{\delta}=R_{\alpha}^{\delta} y^{\delta}$ is called

(i) optimal on the set $M_{\varphi, E}$ if $\left\|x_{\alpha}^{\delta}-x^{\dagger}\right\| \leq E \sqrt{\rho^{-1}\left(\frac{\delta^{2}}{E^{2}}\right)}$

(ii) order optimal on the set $M_{\varphi, E}$ if $\left\|x_{\alpha}^{\delta}-x^{\dagger}\right\| \leq c E \sqrt{\rho^{-1}\left(\frac{\delta^{2}}{E^{2}}\right)}$ with $c \geq 1$.

In [13] it has been proved that there exist special regularization methods that are optimal on the set $M_{\varphi, E}$. One of these methods is the method of generalized Tikhonov regularization. In this method a regularized approximation 
$x_{\alpha}^{\delta}$ is determined by solving the minimization problem

$$
\begin{aligned}
& \min _{x \in X} J_{\alpha}(x) \\
& J_{\alpha}(x)=\left\|A x-y^{\delta}\right\|^{2}+\alpha\left\|\left[\varphi\left(A^{*} A\right)\right]^{-1 / 2} x\right\|^{2} \quad(x \in X)
\end{aligned}
$$

or, equivalently, by solving the Euler equation

$$
\left(A^{*} A+\alpha\left[\varphi\left(A^{*} A\right)\right]^{-1}\right) x_{\alpha}^{\delta}=A^{*} y^{\delta}
$$

of Tikhonov's functional $J_{\alpha}$. This method is optimal on the set $M_{\varphi, E}$ given by (1.4) provided the regularization parameter $\alpha$ is chosen properly. In fact, we have the following result from [13]:

Theorem 2.3. Let $M_{\varphi, E}$ be given by (1.4), let Assumption 1.1 be satisfied, $\varphi$ be twice differentiable, $\rho$ be strictly convex and $\frac{\delta^{2}}{E^{2}} \leq a \varphi(a)$. Then the Tikhonov regularized approximation $x_{\alpha}^{\delta}$ defined by $(2.3)$ is optimal on $M_{\varphi, E}$ provided the regularization parameter $\alpha$ is chosen by

$$
\alpha=\frac{\lambda}{\varphi^{-1}(\lambda) \varphi^{\prime}\left(\varphi^{-1}(\lambda)\right)}\left(\frac{\delta}{E}\right)^{2} \quad \text { with } \quad \lambda=\rho^{-1}\left(\frac{\delta^{2}}{E^{2}}\right) .
$$

It is to be mentioned that, for method (2.3), in a general setting of an unbounded operator $L$ in place of $\left[\varphi\left(A^{*} A\right)\right]^{-\frac{1}{2}}$, order optimal results are obtained by Mair [7] under an apriori choice of the parameter, and by Nair [9] by using the Morozov discrepancy principle.

\section{The general regularization scheme}

The construction of regularized approximations that are optimal on the source set $M_{\varphi, E}$ given in (1.4), such as the ones considered in $[7,9,13]$, requires the knowledge of the function $\varphi$. In practice, however, the smoothness properties of the unknown solution $x^{\dagger}$ of problem (1.1) are generally unknown. Hence, there arises the question if there are regularization methods which do not require the knowledge of the function $\varphi$ and which are order optimal on the set $M_{\varphi, E}$.

We will prove in this section that if the function $\varphi$ is concave, then the classical regularization methods such as Tikhonov regularization, iterated Tikhonov regularization, asymptotical regularization, regularized singular value decomposition and others provide, combined with Morozov's discrepancy principle, regularized approximations $x_{\alpha}^{\delta}$ which are order optimal on the set $M_{\varphi, E}$. 
It is well known that in the classical regularization methods the regularized approximations $x_{\alpha}^{\delta}$ can be represented in the general form

$$
x_{\alpha}^{\delta}=g_{\alpha}\left(A^{*} A\right) A^{*} y^{\delta}
$$

with some piecewise continuous function $g_{\alpha}:(0, a] \rightarrow(0, \infty)$ with $a \geq\|A\|^{2}$ and $\alpha>0$ so that $g_{\alpha}\left(A^{*} A\right)=\int_{0}^{a} g_{\alpha}(\lambda) d E_{\lambda}$. This representation appears to be useful in the theoretical study of regularization methods. For choosing the regularization parameter $\alpha$ we consider Morozov's discrepancy principle (cf. $[8])$. In this principle the regularization parameter $\alpha$ is chosen as the solution of the equation

$$
d(\alpha):=\left\|A x_{\alpha}^{\delta}-y^{\delta}\right\|=C \delta \quad \text { with } \quad C \geq 1
$$

For studying properties of the function $d$ we exploit

Assumption 3.1. The function $g_{\alpha}:(0, a] \rightarrow(0, \infty)$ with $a \geq\|A\|^{2}$ and $\alpha>0$ satisfies

(i) $\sup _{0 \leq \lambda \leq a}\left|g_{\alpha}(\lambda)\right| \leq \frac{\gamma}{\alpha}$ for a constant $\gamma>0$

(ii) $\sup _{0 \leq \lambda \leq a}\left|1-\lambda g_{\alpha}(\lambda)\right| \leq 1$

(iii) $1-\lambda g_{\alpha}(\lambda) \rightarrow 0$ for $\alpha \rightarrow 0$ and all $\lambda \in[0, a]$

(iv) $\left|1-\lambda g_{\alpha_{1}}(\lambda)\right| \leq\left|1-\lambda g_{\alpha_{2}}(\lambda)\right|$ for $\alpha_{1} \leq \alpha_{2}$

(v) $g_{\alpha_{n}}(\lambda) \rightarrow g_{\alpha}(\lambda)$ for $\alpha_{n} \rightarrow \alpha>0$ and all $\lambda \in[0, a]$.

From [15: p. 64, Lemma 3.1] we have that under conditions (i) - (iii) of Assumption 3.1 there hold the limit relations

$$
\lim _{\alpha \rightarrow 0} d(\alpha)=\left\|Q y^{\delta}\right\| \quad \text { and } \quad \lim _{\alpha \rightarrow \infty} d(\alpha)=\left\|y^{\delta}\right\|
$$

where $Q$ denotes the orthoprojection of $Y$ onto $N\left(A^{*}\right)=\overline{R(A)} \perp$. In addition we have from [15: p. 64, Lemma 3.1] that under conditions (iv) $-(\mathrm{v})$ of Assumption 3.1 the function $d$ is monotonically increasing and continuous. Hence, under Assumption 3.1 the nonlinear scalar equation (3.2) possesses a solution $\alpha=\alpha_{D}$ provided

$$
\left\|Q y^{\delta}\right\|<C \delta<\left\|y^{\delta}\right\|
$$

Note that assumption (3.3) on $y^{\delta}$ is not unrealistic. Indeed, since $y \in$ $R(A)$, we have $Q y=0$ so that $\left\|Q y^{\delta}\right\|=\left\|Q\left(y-y^{\delta}\right)\right\| \leq\left\|y-y^{\delta}\right\| \leq \delta$. Hence, condition (3.3) is satisfied, e.g., if $C>1$ and $C \delta<\left\|y^{\delta}\right\|$.

For our further study we exploit some assumption from [15: p. 75]: 
Assumption 3.2. The function $g_{\alpha}:(0, a] \rightarrow(0, \infty)$ with $a \geq\|A\|^{2}$ and $\alpha>0$ satisfies

(i) $g_{\alpha}(\lambda) \geq 0$

(ii) $0 \leq 1-\lambda g_{\alpha}(\lambda) \leq \frac{g_{\alpha}(\lambda)}{\kappa_{\alpha}}$ with $\kappa_{\alpha}:=\sup _{0 \leq \lambda \leq a} g_{\alpha}(\lambda)$

(iii) $\frac{\beta}{\alpha} \leq \kappa_{\alpha} \leq \frac{\gamma}{\alpha}$ with constants $\beta>0$ and $\gamma>0$.

In our next theorem we provide order optimal error bounds for $\left\|x_{\alpha}^{\delta}-x^{\dagger}\right\|$. We shall make use of the relation

$$
\begin{gathered}
\left\|x_{\alpha}^{\delta}-x^{\dagger}\right\|^{2}+\kappa_{\alpha}\left\{\left\|A x_{\alpha}^{\delta}-y^{\delta}\right\|^{2}-\left\|A x^{\dagger}-y^{\delta}\right\|^{2}\right\} \\
\leq\left(\left[I-A^{*} A g_{\alpha}\left(A^{*} A\right)\right] x^{\dagger}, x^{\dagger}\right)
\end{gathered}
$$

which holds true under Assumption 3.2 (see [15: p. 77/Lemma 4.1]).

Theorem 3.3. Let $M_{\varphi, E}$ be given by (1.4), let Assumption 1.1 be satisfied and let $x_{\alpha}^{\delta}$ be the regularized approximation (3.1) with $g_{\alpha}$ satisfying Assumption 3.2. Let Assumption 3.1 and (3.3) hold and let $\alpha$ be chosen by Morozov's discrepancy principle (3.2). If the function $\varphi$ is concave, then $x_{\alpha}^{\delta}$ is order optimal on the set $M_{\varphi, E}$ and

$$
\left\|x_{\alpha}^{\delta}-x^{\dagger}\right\| \leq(C+1) E \sqrt{\rho^{-1}\left(\frac{\delta^{2}}{E^{2}}\right)} .
$$

Proof. For all $(\alpha, \lambda)$ for which $g_{\alpha}$ is defined we define

$$
r_{\alpha}(\lambda)=1-\lambda g_{\alpha}(\lambda)
$$

Let $\alpha=\alpha_{D}$ be the regularization parameter chosen by (3.2). Due to relation (3.4) and to

$$
\begin{aligned}
\left\|A x^{\dagger}-y^{\delta}\right\| & \leq\left\|A x_{\alpha}^{\delta}-y^{\delta}\right\| \\
\left(\left[I-A^{*} A g_{\alpha}\left(A^{*} A\right)\right] x^{\dagger}, x^{\dagger}\right) & =\left\|\left[r_{\alpha}\left(A^{*} A\right)\right]^{\frac{1}{2}} x^{\dagger}\right\|^{2}
\end{aligned}
$$

we have

$$
\left\|x_{\alpha}^{\delta}-x^{\dagger}\right\| \leq\left\|\left[r_{\alpha}\left(A^{*} A\right)\right]^{\frac{1}{2}} x^{\dagger}\right\| .
$$

Since $\varphi$ is a concave function with $\lim _{\lambda \rightarrow 0} \varphi(\lambda)=0$ we have $t \varphi(\lambda) \leq \varphi(t \lambda)$ for $t \in[0,1]$ or, equivalently, $\varphi^{-1}(t \varphi(\lambda)) \leq \lambda t$ which, due to $\rho(\lambda):=\lambda \varphi^{-1}(\lambda)$, provides the relation $\rho(t \varphi(\lambda)) \leq t^{2} \lambda \varphi(\lambda)$. We apply this estimate with $t=$ $r_{\alpha}(\lambda):=1-\lambda g_{\alpha}(\lambda)$ and obtain

$$
\rho\left(r_{\alpha}(\lambda) \varphi(\lambda)\right) \leq \lambda r_{\alpha}^{2}(\lambda) \varphi(\lambda)
$$

Let $x_{\alpha}$ be approximation (3.1) with $y^{\delta}$ replaced by the exact data $y$, that is,

$$
x_{\alpha}=g_{\alpha}\left(A^{*} A\right) A^{*} y .
$$


Since $\alpha=\alpha_{D}$ is the solution of equation (3.2), we have

$$
\begin{aligned}
\left\|A x_{\alpha}-A x^{\dagger}\right\| & =\left\|A r_{\alpha}\left(A^{*} A\right) x^{\dagger}\right\| \\
& =\left\|r_{\alpha}\left(A A^{*}\right) A x^{\dagger}\right\| \\
& \leq\left\|r_{\alpha}\left(A A^{*}\right) y^{\delta}\right\|+\left\|r_{\alpha}\left(A A^{*}\right)\left(A x^{\dagger}-y^{\delta}\right)\right\| \\
& =\left\|A x_{\alpha}^{\delta}-y^{\delta}\right\|+\left\|r_{\alpha}\left(A A^{*}\right)\left(A x^{\dagger}-y^{\delta}\right)\right\| \\
& \leq(C+1) \delta .
\end{aligned}
$$

Now we exploit (1.4), the convexity of $\rho$, inequalities (3.6) - (3.7) and Jensen's inequality. Thus

$$
\begin{aligned}
\rho\left(\frac{\left.\| r_{\alpha}\left(A^{*} A\right)\right]^{\frac{1}{2}} x^{\dagger} \|^{2}}{\|v\|^{2}}\right) & =\rho\left(\frac{\int_{0}^{a} r_{\alpha}(\lambda) \varphi(\lambda) d\left\|E_{\lambda} v\right\|^{2}}{\int_{0}^{a} d\left\|E_{\lambda} v\right\|^{2}}\right) \\
& \leq \frac{\int_{0}^{a} \rho\left(r_{\alpha}(\lambda) \varphi(\lambda)\right) d\left\|E_{\lambda} v\right\|^{2}}{\int_{0}^{a} d\left\|E_{\lambda} v\right\|^{2}} \\
& \leq \frac{\int_{0}^{a} \lambda r_{\alpha}^{2}(\lambda) \varphi(\lambda) d\left\|E_{\lambda} v\right\|^{2}}{\int_{0}^{a} d\left\|E_{\lambda} v\right\|^{2}} \\
& =\frac{\left\|\left(A^{*} A\right)^{\frac{1}{2}} r_{\alpha}\left(A^{*} A\right)\left[\varphi\left(A^{*} A\right)\right]^{\frac{1}{2}} v\right\|^{2}}{\|v\|^{2}} \\
& =\frac{\left\|A x_{\alpha}-A x^{\dagger}\right\|^{2}}{\|v\|^{2}} \\
& \leq \frac{(C+1)^{2} \delta^{2}}{\|v\|^{2}} .
\end{aligned}
$$

Using the monotonicity of $\varphi^{-1}$ and the realtions $\varphi^{-1}(\lambda)=\frac{1}{\lambda} \rho(\lambda)$ and (3.10), we obtain

$$
\begin{aligned}
\varphi^{-1}\left(\frac{\left\|\left[r_{\alpha}\left(A^{*} A\right)\right]^{\frac{1}{2}} x^{\dagger}\right\|^{2}}{(C+1)^{2} E^{2}}\right) & \leq \varphi^{-1}\left(\frac{\left\|\left[r_{\alpha}\left(A^{*} A\right)\right]^{\frac{1}{2}} x^{\dagger}\right\|^{2}}{\|v\|^{2}}\right) \\
& =\frac{\|v\|^{2}}{\left\|\left[r_{\alpha}\left(A^{*} A\right)\right]^{1 / 2} x^{\dagger}\right\|^{2}} \rho\left(\frac{\left\|\left[r_{\alpha}\left(A^{*} A\right)\right]^{\frac{1}{2}} x^{\dagger}\right\|^{2}}{\|v\|^{2}}\right) \\
& \leq \frac{(C+1)^{2} \delta^{2}}{\left\|\left[r_{\alpha}\left(A^{*} A\right)\right]^{\frac{1}{2}} x^{\dagger}\right\|^{2}}
\end{aligned}
$$

or, equivalently,

$$
\rho\left(\frac{\left\|\left[r_{\alpha}\left(A^{*} A\right)\right]^{\frac{1}{2}} x^{\dagger}\right\|^{2}}{(C+1)^{2} E^{2}}\right) \leq \frac{\delta^{2}}{E^{2}} .
$$

This estimate together with (3.6) leads to (3.5) 
Let us discuss two special cases. In our first special case we consider operator equations with finitely smoothing operators $A$ and assume that $x^{\dagger} \in$ $M_{p, E}$ with $M_{p, E}$ given in (1.2). This set has form (1.4) with $\varphi(\lambda)=\lambda^{p}$. For this function Assumption 1.1 is satisfied. Since $\varphi^{-1}(\lambda)=\lambda^{\frac{1}{p}}$ we obtain $\rho(\lambda):=\lambda \varphi^{-1}(\lambda)=\lambda^{\frac{p+1}{p}}$, consequently, $\rho^{-1}(\lambda)=\lambda^{\frac{p}{p+1}}$. Since $\varphi(\lambda)=\lambda^{p}$ is concave for $p \leq 1$, the following result is a special case of Theorem 3.3.

Corollary 3.4. Let $x_{\alpha}^{\delta}$ be the regularized approximation (3.1) with $g_{\alpha}$ satisfying Assumption 3.2. Let Assumption 3.1 and (3.3) hold and let $\alpha$ be chosen by Morozov's discrepancy principle (3.2). If $p \leq 1$, then $x_{\alpha}^{\delta}$ is order optimal on the set $M_{p, E}$ given in (1.2), and

$$
\left\|x_{\alpha}^{\delta}-x^{\dagger}\right\| \leq(C+1) E^{\frac{1}{p+1}} \delta^{\frac{p}{p+1}} .
$$

Although this classical result of Corollary 3.4 is not new (compare [15: p. $78 /$ Theorem 4.2]), we realize that it can be obtained as a special case of our more general Theorem 3.1.

In our second special case we consider operator equations with infinitely smoothing operators $A$ and assume that $x^{\dagger} \in M_{p, E}^{\log }$ with $M_{p, E}^{\log }$ given in (1.3). This set has form (1.4) with $\varphi(\lambda)=\left[\ln \frac{1}{\lambda}\right]^{-p}$. For this function Assumption 1.1 is satisfied. Since $\varphi^{-1}(\lambda)=e^{-1 / \lambda^{1 / p}}$ we obtain $\rho(\lambda):=\lambda \varphi^{-1}(\lambda)=$ $\lambda e^{-1 / \lambda^{1 / p}}$, consequently,

$$
\rho^{-1}(\lambda)=\left[\ln \frac{1}{\lambda}\right]^{-p}(1+o(1)) \quad \text { for } \quad \lambda \rightarrow 0
$$

(see, e.g., [7]). Since $\varphi(\lambda)$ is concave for $\lambda \leq \frac{1}{(p+1)^{p}}$, from Theorem 3.3 we obtain

Corollary 3.5. Let $x_{\alpha}^{\delta}$ be the regularized approximation (3.1) with $g_{\alpha}$ satisfying Assumption 3.2. Let Assumption 3.1 and (3.3) hold and let $\alpha$ be chosen by Morozov's discrepancy principle (3.2). If $\|A\| \leq \frac{1}{(p+1)^{p / 2}}$, then $x_{\alpha}^{\delta}$ is order optimal on the set $M_{p, E}^{\log }$ given in (1.3) and

$$
\left\|x_{\alpha}^{\delta}-x^{\dagger}\right\| \leq(C+1) E\left[\ln \frac{E^{2}}{\delta^{2}}\right]^{-\frac{p}{2}}(1+o(1)) \quad \text { for } \delta \rightarrow 0 .
$$

Remarks. For Tikhonov regularization, a result analogous to Corollary 3.5 has been proved by Pereverzev and Schock [11] under a modified form of Morozov's discrepancy principle. For the general regularization of form (3.1), Hohage [5] proved a result analogous to Corollary 3.5 under the assumption on $g_{\alpha}$ that $\lambda^{\mu}\left[1-\lambda g_{\alpha}(\lambda)\right] \leq c_{\mu} \alpha^{\mu}$ for $0 \leq \mu \leq \mu_{0}$ for some $\mu_{0}>0$. We 
deduced Corollary 3.5 from a more general result, Theorem 3.3, whose proof also seems to be much simpler than the procedures adopted in $[5,11]$.

While proving Theorem 3.3 we made use of relation (3.4). For the special case of Tikhonov regularization we shall provide in Section 5 an alternative proof which does not require (3.4) and allows additional discretization.

\section{The general regularization scheme revisited}

Theorem 3.3 requires the assumption that $\varphi$ is concave. This assumption is too strong for certain special regularization methods and certain special source sets. Therefore we reconsider the general regularization scheme under some other assumption which appears to be weaker in certain circumstances. However, let us start our studies with some general order optimality result for the regularization error $\left\|x_{\alpha}-x^{\dagger}\right\|$.

Proposition 4.1. Let $M_{\varphi, E}$ be given by (1.4) and let $x_{\alpha}$ be the regularized approximation (3.8). Let Assumption 3.1 and (3.3) hold and let $\alpha$ be chosen by Morozov's discrepancy principle (3.2). If $x^{\dagger} \in M_{\varphi, E}$, then

$$
\left\|x_{\alpha}-x^{\dagger}\right\| \leq(C+1) \omega\left(\delta, M_{\varphi, E}\right)
$$

with $\omega\left(\delta, M_{\varphi, E}\right)$ given by (2.1). If in addition Assumption 1.1 is satisfied, then

$$
\left\|x_{\alpha}-x^{\dagger}\right\| \leq(C+1) E \sqrt{\rho^{-1}\left(\frac{\delta^{2}}{E^{2}}\right)} .
$$

Proof. We define $z_{\alpha}=\frac{1}{C+1}\left(x_{\alpha}-x^{\dagger}\right)$ with $\alpha$ chosen by (3.2). Due to

$$
x_{\alpha}-x^{\dagger}=\left[g_{\alpha}\left(A^{*} A\right) A^{*} A-I\right]\left[\varphi\left(A^{*} A\right)\right]^{\frac{1}{2}} v
$$

we have $z_{\alpha}=\left[\varphi\left(A^{*} A\right)\right]^{\frac{1}{2}} w$, where $w=\frac{1}{C+1}\left[g_{\alpha}\left(A^{*} A\right) A^{*} A-I\right] v$ satisfies

$$
\|w\| \leq \frac{1}{C+1}\|v\| \leq E
$$

Hence, $z_{\alpha} \in M_{\varphi, E}$. In addition, due to (3.9) we have $\left\|A z_{\alpha}\right\| \leq \delta$. Hence, due to (2.1) we obtain $\left\|z_{\alpha}\right\| \leq \omega\left(\delta, M_{\varphi, E}\right)$ or, equivalently, (4.1). Now (4.2) follows from (4.1) and (2.2)

Our next proposition provides an order optimality result under the condition that $\alpha_{D} \geq \alpha_{0}$ with $\alpha_{D}$ as the solution of equation (3.2) and $\alpha_{0}$ given by

$$
\alpha_{0}=\varphi^{-1}\left(\rho^{-1}\left(\frac{\delta^{2}}{E^{2}}\right)\right)
$$


This order optimality result requires an additional assumption on $g_{\alpha}$ that there exists some constant $c_{0}$ such that

$$
\sup _{\lambda \in[0, a]}\left|\sqrt{\lambda} g_{\alpha}(\lambda)\right| \leq \frac{c_{0}}{\sqrt{\alpha}}
$$

Proposition 4.2. Let $M_{\varphi, E}$ be given by (1.4), let Assumption 1.1 be satisfied and let $x_{\alpha}^{\delta}$ be the regularized approximation (3.1). Let Assumption 3.1 and (3.3) hold, let $\alpha_{D}$ be the solution of (3.2) and let $\alpha_{0}$ be given by (4.3). If $\alpha_{D} \geq \alpha_{0}$, then $x_{\alpha_{D}}^{\delta}$ is order optimal on the set $M_{\varphi, E}$, and

$$
\left\|x_{\alpha_{D}}^{\delta}-x^{\dagger}\right\| \leq\left(C+c_{0}+1\right) E \sqrt{\rho^{-1}\left(\frac{\delta^{2}}{E^{2}}\right)} .
$$

Proof. Consider $x_{\alpha}$ given by (3.8). Due to (4.4) we have

$$
\left\|x_{\alpha}^{\delta}-x_{\alpha}\right\|=\left\|g_{\alpha}\left(A^{*} A\right) A^{*}\left(y^{\delta}-y\right)\right\| \leq \frac{c_{0} \delta}{\sqrt{\alpha}}
$$

From (4.6) and $\alpha_{D} \geq \alpha_{0}$ we obtain

$$
\left\|x_{\alpha_{D}}^{\delta}-x_{\alpha_{D}}\right\| \leq \frac{c_{0} \delta}{\sqrt{\alpha_{D}}} \leq \frac{c_{0} \delta}{\sqrt{\alpha_{0}}}=c_{0} E \sqrt{\rho^{-1}\left(\frac{\delta^{2}}{E^{2}}\right)}
$$

Consequently, (4.5) follows from (4.2) and the triangle inequality

Now it remains to study the case $\alpha_{D} \leq \alpha_{0}$. In this case, however, we need the additional condition that there exists some constant $c_{\varphi}$ such that

$$
\sup _{0<\lambda \leq a}\left|\left[1-\lambda g_{\alpha}(\lambda)\right] \varphi(\lambda)\right| \leq c_{\varphi} \varphi(\alpha)
$$

Theorem 4.3. Let $M_{\varphi, E}$ be given by (1.4) and let Assumptions 1.1 be satisfied. Let $x_{\alpha}^{\delta}$ be the regularized approximation (3.1) with $g_{\alpha}$ satisfying Assumption 3.2, (4.4) and (4.7). Let in addition Assumption 3.1 and (3.3) hold and let $\alpha_{D}$ be the solution of equation (3.2). Then $x_{\alpha_{D}}^{\delta}$ is order optimal on the set $M_{\varphi, E}$ and

$$
\left\|x_{\alpha_{D}}^{\delta}-x^{\dagger}\right\| \leq c E \sqrt{\rho^{-1}\left(\frac{\delta^{2}}{E^{2}}\right)}
$$

with $c=\max \left\{C+c_{0}+1, \sqrt{c_{\varphi}}\right\}$.

Proof. Let us distinguish two cases. In the first case of $\alpha_{D} \geq \alpha_{0}$ with $\alpha_{0}$ given by (4.3) the proof of this theorem follows from Proposition 4.2. 
It remains to consider the second case $\alpha_{D} \leq \alpha_{0}$. Using relation (3.4) and exploiting (1.4) and (4.7) we obtain

$$
\begin{aligned}
\left\|x_{\alpha_{D}}^{\delta}-x^{\dagger}\right\|^{2} & \leq\left(\left[I-g_{\alpha_{D}}\left(A^{*} A\right) A^{*} A\right] \varphi\left(A^{*} A\right) v, v\right) \\
& \leq E^{2} \sup _{0<\lambda \leq a}\left|\left[1-\lambda g_{\alpha_{D}}(\lambda)\right] \varphi(\lambda)\right| \\
& \leq E^{2} c_{\varphi} \varphi\left(\alpha_{D}\right) .
\end{aligned}
$$

Hence, since $\alpha_{D} \leq \alpha_{0}$ and $\varphi$ is monotonically increasing, we have in the second case

$$
\left\|x_{\alpha_{D}}^{\delta}-x^{\dagger}\right\|^{2} \leq E^{2} c_{\varphi} \varphi\left(\alpha_{0}\right)=E^{2} c_{\varphi} \rho^{-1}\left(\frac{\delta^{2}}{E^{2}}\right) .
$$

This completes the proof

The proof of the following corollary, a companion result to Corollary 3.4, is immediate from Theorem 4.3.

Corollary 4.4. Let $x_{\alpha}^{\delta}$ be the regularized approximation (3.1) with $g_{\alpha}$ satisfying Assumption 3.2 and (4.4). Let Assumption 3.1 and (3.3) hold and let $\alpha$ be chosen by Morozov's discrepancy principle (3.2). Suppose, in addition, that there exists $p_{0}>0$ with the property that for each $p \in\left(0, p_{0}\right]$ there exists $c_{p}>0$ such that

$$
\sup _{0<\lambda \leq a} \lambda^{p}\left[1-\lambda g_{\alpha}(\lambda)\right] \leq c_{p} \alpha^{p} \quad \text { for } \quad 0<p \leq p_{0} .
$$

Then for each $p \in\left(0, p_{0}\right], x_{\alpha}^{\delta}$ is order optimal on the set $M_{p, E}$ given in (1.2) and

$$
\left\|x_{\alpha}^{\delta}-x^{\dagger}\right\| \leq c E^{\frac{1}{p+1}} \delta^{\frac{p}{p+1}}
$$

with $c=\max \left\{C+c_{0}+1, \sqrt{c_{p}}\right\}$.

Our next proposition shows that for many regularizaton methods condition (4.7) in Theorem 4.3 is indeed weaker than the concavity condition for the function $\varphi$ in Theorem 3.3.

Proposition 4.5. Let Assumption 1.1 be satisfied. Define

$$
h(\lambda):=1-\alpha \lambda g_{\alpha}(\alpha \lambda) \quad(0 \leq \lambda<\infty)
$$

and assume that $h$ is independent of $\alpha$, that $0 \leq h(\lambda) \leq 1$ and that $\lambda h(\lambda) \leq 1$. If the function $\varphi$ is concave, then assumption (4.7) holds true with $c_{\varphi}=1$.

Proof. We substitute $\lambda=\alpha s$ into (4.7) and obtain from (4.10)

$$
\sup _{\lambda \in[0, a]}\left|\left[1-\lambda g_{\alpha}(\lambda)\right] \varphi(\lambda)\right| \leq \sup _{s \geq 0}\left|\left[1-\alpha s g_{\alpha}(\alpha s)\right] \varphi(\alpha s)\right|=\sup _{s \geq 0}|h(s) \varphi(\alpha s)| .
$$


Since $\varphi$ is a concave function with $\lim _{\lambda \rightarrow 0} \varphi(\lambda)=0$ we have $t \varphi(s) \leq \varphi(t s)$ for $t \in[0,1]$. We apply this estimate with $t=h(s)$ and obtain

$$
h(s) \varphi(\alpha s) \leq \varphi(\alpha s h(s)) .
$$

Since $\varphi$ is monotonically increasing and $\operatorname{sh}(s) \leq 1$ there follows

$$
h(s) \varphi(\alpha s) \leq \varphi(\alpha s h(s)) \leq \varphi(\alpha) .
$$

This estimate and (4.11) provide the result of the proposition

Examples for regularization methods (3.1) with $h$ defined in (4.10) satisfying $0 \leq h(\lambda) \leq 1$ and $\lambda h(\lambda) \leq 1$ include, e.g.,

(a) the method of ordinary Tikhonov regularization with $h(\lambda)=\frac{1}{\lambda+1}$

(b) the method of asymptotical regularization with $h(\lambda)=e^{-\lambda}$

(c) the method of truncated singular value decomposition with $h(\lambda)=0$ for $\lambda \geq 1$ and $h(\lambda)=1-\lambda$ for $\lambda \leq 1$.

\section{Discretization in Tikhonov's method}

In the method of ordinary Tikhonov regularization the regularized approximation $x_{\alpha}^{\delta}$ is determined by solving the minimization problem

$$
\begin{aligned}
& \min _{x \in X} J_{\alpha}(x) \\
& J_{\alpha}(x)=\left\|A x-y^{\delta}\right\|^{2}+\alpha\|x\|^{2} \quad(x \in X)
\end{aligned}
$$

or, equivalently, $x_{\alpha}^{\delta}$ is the unique solution of the Euler equation

$$
\left(A^{*} A+\alpha I\right) x_{\alpha}^{\delta}=A^{*} y^{\delta}
$$

of Tikhonov's functional $J_{\alpha}$. Hence, $x_{\alpha}^{\delta}$ has form (3.1) with $g_{\alpha}(\lambda)=\frac{1}{\lambda+\alpha}$. The computation of regularized approximations $x_{\alpha}^{\delta}$ requires the numerical realization of (3.1) and (3.2) in finite-dimensional spaces. Let us approximate the space $X$ by a finite-dimensional subspace $X_{n}$. Then, in Tikhonov's method a finite-dimensional regularized approximation $x_{n, \alpha}^{\delta}$ can be defined by solving the finite-dimensional minimization problem

$$
\begin{aligned}
& \min _{x \in X_{n}} J_{\alpha}(x) \\
& J_{\alpha}(x)=\left\|A x-y^{\delta}\right\|^{2}+\alpha\|x\|^{2} \quad\left(x \in X_{n}\right) .
\end{aligned}
$$

Problem (5.1) is equivalent to minimizing the functional $\left\|A_{n} x-y^{\delta}\right\|^{2}+\alpha\|x\|^{2}$ over $X$, where $A_{n}=A P_{n}$ and $P_{n}: X \rightarrow X$ is the orthogonal projector onto 
the subspace $X_{n}$. Therefore, the finite-dimensional regularized approximation $x_{n, \alpha}^{\delta}$ of problem (5.1) is given by

$$
x_{n, \alpha}^{\delta}=\left(A_{n}^{*} A_{n}+\alpha I\right)^{-1} A_{n}^{*} y^{\delta}
$$

where $A_{n}=A P_{n}$. If $\left\{\phi_{1}, \ldots, \phi_{n}\right\}$ is a basis of $X_{n}$, then (5.2) has the form

$$
x_{n, \alpha}^{\delta}=\sum_{j=1}^{n} c_{j} \phi_{j}
$$

with $c=\left(c_{1}, \ldots, c_{n}\right)^{T} \in \mathbb{R}^{n}$ as the unique solution of the linear system of equations

$$
[\mathbf{A}+\alpha \mathbf{\Phi}] c=d
$$

where $\mathbf{A}$ and $\boldsymbol{\Phi}$ are $n \times n$ matrices with $i j$-th entries $\left(A \phi_{j}, A \phi_{i}\right)$ and $\left(\phi_{j}, \phi_{j}\right)$, respectively, and $d \in \mathbb{R}^{n}$ is a vector with entries $d_{i}=\left(y^{\delta}, A \phi_{i}\right)$.

For choosing the regularization parameter we consider a discretized discrepancy principle, that is, we choose $\alpha$ as the solution of the equation

$$
d_{n}(\alpha):=\left\|A x_{n, \alpha}^{\delta}-y^{\delta}\right\|=\left(1+\varepsilon_{n}\right) \delta
$$

with some constant $\varepsilon_{n} \geq 0$. The function $d_{n}$ is continuous with $d_{n}(0)=$ $\left\|Q_{n} y^{\delta}\right\|$ and $\lim _{\alpha \rightarrow \infty} d_{n}(\bar{\alpha})=\left\|y^{\delta}\right\|$ where $Q_{n}$ denotes the orthoprojection of $Y$ onto $N\left(A_{n}^{*}\right)=\overline{R\left(A_{n}\right)} \perp$. Hence, the nonlinear scalar equation (5.3) possesses a unique solution $\alpha_{D}$ provided

$$
\left\|Q_{n} y^{\delta}\right\|<\left(1+\varepsilon_{n}\right) \delta<\left\|y^{\delta}\right\|
$$

In our next theorem we show that our finite-dimensional regularized approximation (5.2) with $\alpha$ chosen by (5.3) is order optimal on the general source set (1.4) provided $\varphi$ is concave and $P_{n}$ satisfies approximation property (5.5).

Theorem 5.1. Let $M_{\varphi, E}$ be given by (1.4) and let Assumption 1.1 be satisfied. Let $x_{n, \alpha}^{\delta}$ the finite-dimensional regularized approximation (5.2), let (5.4) hold and let $\alpha$ be chosen by the discretized discrepancy principle (5.3). If $P_{n}$ satisfies

$$
\left\|A\left(I-P_{n}\right) x^{\dagger}\right\| \leq \varepsilon_{n} \delta
$$

and if $\varphi$ is concave, then $x_{n, \alpha}^{\delta}$ is order optimal on the set $M_{\varphi, E}$ and

$$
\left\|x_{n, \alpha}^{\delta}-x^{\dagger}\right\| \leq\left(2+\varepsilon_{n}\right) E \sqrt{\rho^{-1}\left(\frac{\delta^{2}}{E^{2}}\right)}
$$


Proof. Since $x_{n, \alpha}^{\delta}$ satisfies the equation $\alpha x_{n, \alpha}^{\delta}=P_{n} A^{*}\left[y^{\delta}-A x_{n, \alpha}^{\delta}\right]$ we obtain due to $A P_{n} x_{n, \alpha}^{\delta}=A x_{n, \alpha}^{\delta}$ that

$$
\begin{aligned}
\left\|x_{n, \alpha}^{\delta}-x^{\dagger}\right\|^{2} & \\
= & \left(x^{\dagger}-x_{n, \alpha}^{\delta}, x^{\dagger}\right)-\left(x^{\dagger}-x_{n, \alpha}^{\delta}, x_{n, \alpha}^{\delta}\right) \\
= & \left(x^{\dagger}-x_{n, \alpha}^{\delta}, x^{\dagger}\right)-\frac{1}{\alpha}\left(x^{\dagger}-x_{n, \alpha}^{\delta}, P_{n} A^{*}\left[y^{\delta}-A x_{n, \alpha}^{\delta}\right]\right) \\
= & \left(x^{\dagger}-x_{n, \alpha}^{\delta}, x^{\dagger}\right)-\frac{1}{\alpha}\left(A P_{n} x^{\dagger}-A x_{n, \alpha}^{\delta}, y^{\delta}-A x_{n, \alpha}^{\delta}\right) \\
= & \left(x^{\dagger}-x_{n, \alpha}^{\delta}, x^{\dagger}\right)+\frac{1}{2 \alpha}\left\{\left\|A P_{n} x^{\dagger}-y^{\delta}\right\|^{2}\right. \\
& \left.-\left\|A x_{n, \alpha}^{\delta}-y^{\delta}\right\|^{2}-\left\|A P_{n} x^{\dagger}-A x_{n, \alpha}^{\delta}\right\|^{2}\right\} .
\end{aligned}
$$

From (5.5) we have

$$
\left\|A P_{n} x^{\dagger}-y^{\delta}\right\| \leq\left\|A x^{\dagger}-y^{\delta}\right\|+\left\|A\left(I-P_{n}\right) x^{\dagger}\right\| \leq\left(1+\varepsilon_{n}\right) \delta .
$$

Consequently, due to (5.7) and (5.3) we obtain for $\alpha=\alpha_{D}$

$$
\left\|x_{n, \alpha}^{\delta}-x^{\dagger}\right\|^{2} \leq\left(x^{\dagger}-x_{n, \alpha}^{\delta}, x^{\dagger}\right)
$$

Exploiting the source condition $x^{\dagger} \in M_{\varphi, E}$ with $M_{\varphi, E}$ given in (1.4) yields

$$
\left\|x_{n, \alpha}^{\delta}-x^{\dagger}\right\|^{2} \leq E\left\|\left[\varphi\left(A^{*} A\right)\right]^{\frac{1}{2}}\left(x_{n, \alpha}^{\delta}-x^{\dagger}\right)\right\|,
$$

hence

$$
\frac{\left\|x_{n, \alpha}^{\delta}-x^{\dagger}\right\|^{2}}{\left(2+\varepsilon_{n}\right)^{2} E^{2}} \leq \frac{\left\|\left[\varphi\left(A^{*} A\right)\right]^{\frac{1}{2}}\left(x_{n, \alpha}^{\delta}-x^{\dagger}\right)\right\|^{2}}{\left\|x_{n, \alpha}^{\delta}-x^{\dagger}\right\|^{2}} .
$$

Since $\varphi$ is monotone and concave we obtain that $\varphi^{-1}$ is monotone and convex. We use the monotonicity of $\varphi^{-1}$, apply Jensen's inequality and obtain

$$
\begin{aligned}
\varphi^{-1}\left(\frac{\left\|x_{n, \alpha}^{\delta}-x^{\dagger}\right\|^{2}}{\left(2+\varepsilon_{n}\right)^{2} E^{2}}\right) & \leq \varphi^{-1}\left(\frac{\left\|\left[\varphi\left(A^{*} A\right)\right]^{\frac{1}{2}}\left(x_{n, \alpha}^{\delta}-x^{\dagger}\right)\right\|^{2}}{\left\|x_{n, \alpha}^{\delta}-x^{\dagger}\right\|^{2}}\right) \\
& =\varphi^{-1}\left(\frac{\int_{0}^{a} \varphi(\lambda) d\left\|E_{\lambda}\left(x_{n, \alpha}^{\delta}-x^{\dagger}\right)\right\|^{2}}{\int_{0}^{a} d\left\|E_{\lambda}\left(x_{n, \alpha}^{\delta}-x^{\dagger}\right)\right\|^{2}}\right) \\
& \leq \frac{\int_{0}^{a} \lambda d\left\|E_{\lambda}\left(x_{n, \alpha}^{\delta}-x^{\dagger}\right)\right\|^{2}}{\int_{0}^{a} d\left\|E_{\lambda}\left(x_{n, \alpha}^{\delta}-x^{\dagger}\right)\right\|^{2}} \\
& =\frac{\left\|A x_{n, \alpha}^{\delta}-A x^{\dagger}\right\|^{2}}{\left\|x_{n, \alpha}^{\delta}-x^{\dagger}\right\|^{2}} .
\end{aligned}
$$


Due to $\lambda \varphi^{-1}(\lambda)=\rho(\lambda)$ and

$$
\left\|A x_{n, \alpha}^{\delta}-A x^{\dagger}\right\| \leq\left\|A x_{n, \alpha}^{\delta}-y^{\delta}\right\|+\left\|A x^{\dagger}-y^{\delta}\right\| \leq\left(2+\varepsilon_{n}\right) \delta
$$

we obtain

$$
\rho\left(\frac{\left\|x_{n, \alpha}^{\delta}-x^{\dagger}\right\|^{2}}{\left(2+\varepsilon_{n}\right)^{2} E^{2}}\right) \leq \frac{\delta^{2}}{E^{2}}
$$

which provides (5.6)

Note that for $P_{n}=I, X_{n}=X$ and $1+\varepsilon_{n}=C$ the results of Theorem 5.1 and Theorem 3.3 with $g_{\alpha}(\lambda)=\frac{1}{\lambda+\alpha}$ coincide.

\section{Asymptotical regularization}

In the method of asymptotical regularization the regularized approximation $x_{\alpha}^{\delta}$ is given by $x_{\alpha}^{\delta}=x\left(\frac{1}{\alpha}\right)$ where $x=x(t)$ is obtained by solving the initial value problem

$$
\left.\begin{array}{rl}
\frac{d}{d t} x(t)+A^{*} A x(t) & =A^{*} y^{\delta} \quad\left(0<t \leq \frac{1}{\alpha}\right) \\
x(0) & =0
\end{array}\right\} .
$$

It is well known that $x_{\alpha}^{\delta}$ has the representation

$$
x_{\alpha}^{\delta}=g_{\alpha}\left(A^{*} A\right) A^{*} y^{\delta} \quad \text { with } \quad g_{\alpha}(\lambda)=\frac{1-e^{-\frac{\lambda}{\alpha}}}{\lambda} .
$$

Obviously, this method fits into the framework of the general regularization method (3.1). In this section we will give, compared with Theorem 4.3, some other proof for order optimal error bounds which instead of assumption (4.7) requires some weaker assumption. We start our studies with providing some monotonicity property.

Proposition 6.1. Let $x_{\alpha}^{\delta}$ be the regularized approximation (6.2). Let (3.3) hold and let $\alpha_{D}$ be the regularization parameter obtained by Morozov's discrepancy principle (3.2). Then

$$
\left\|x_{\alpha_{D}}^{\delta}-x^{\dagger}\right\| \leq\left\|x_{\alpha}^{\delta}-x^{\dagger}\right\| \quad \text { for } \quad \alpha_{D} \leq \alpha .
$$

Proof. From $g_{\alpha}(\lambda)=\frac{1-e^{-\frac{\lambda}{\alpha}}}{\lambda}$ we have

$$
\frac{d}{d \alpha} g_{\alpha}(\lambda)=-\frac{1}{\alpha^{2}} e^{-\frac{\lambda}{\alpha}}=\frac{1}{\alpha^{2}}\left(\lambda g_{\alpha}(\lambda)-1\right) .
$$


Consequently,

$$
\frac{d}{d \alpha} g_{\alpha}\left(A A^{*}\right) y^{\delta}=\frac{1}{\alpha^{2}}\left[A A^{*} g_{\alpha}\left(A A^{*}\right)-I\right] y^{\delta}=\frac{1}{\alpha^{2}}\left[A x_{\alpha}^{\delta}-y^{\delta}\right] .
$$

This identity provides the estimate

$$
\begin{aligned}
\frac{1}{2} \frac{d}{d \alpha}\left\|x_{\alpha}^{\delta}-x^{\dagger}\right\|^{2} & =\left(x_{\alpha}^{\delta}-x^{\dagger}, A^{*} \frac{d}{d \alpha} g_{\alpha}\left(A A^{*}\right) y^{\delta}\right) \\
& =\left(A x_{\alpha}^{\delta}-y^{\delta}+\left(y^{\delta}-y\right), \frac{d}{d \alpha} g_{\alpha}\left(A A^{*}\right) y^{\delta}\right) \\
& =\frac{1}{\alpha^{2}}\left(A x_{\alpha}^{\delta}-y^{\delta}+\left(y^{\delta}-y\right), A x_{\alpha}^{\delta}-y^{\delta}\right) \\
& \geq \frac{\left\|A x_{\alpha}^{\delta}-y^{\delta}\right\|}{\alpha^{2}}\left\{\left\|A x_{\alpha}^{\delta}-y^{\delta}\right\|-\delta\right\} .
\end{aligned}
$$

From this estimate and the monotonicity of the norm $\left\|A x_{\alpha}^{\delta}-y^{\delta}\right\|$ as function of $\alpha$ it follows that, for all $\alpha \geq \alpha_{D}$,

$$
\frac{1}{2} \frac{d}{d \alpha}\left\|x_{\alpha}^{\delta}-x^{\dagger}\right\|^{2} \geq \frac{C \delta}{\alpha_{D}^{2}}(C-1) \delta \geq 0
$$

Thus, we obtain the required property (6.3)

In order to derive order optimal error bounds for $\left\|x_{\alpha_{D}}^{\delta}-x^{\dagger}\right\|$ we distinguish as in Section 4 two cases, a first case with $\alpha_{D} \geq \alpha_{0}$ and a second one with $\alpha_{D} \leq \alpha_{0}$ where $\alpha_{0}$ is given by (4.3). In the first case we will exploit Proposition 4.2 , and in the second case Proposition 6.1. The second case requires, instead of assumption (4.7), the weaker condition that there exists some constant $d_{\varphi}$ such that

$$
\sup _{0<\lambda \leq a}\left|\left[1-\lambda g_{\alpha}(\lambda)\right] \sqrt{\varphi(\lambda)}\right| \leq d_{\varphi} \sqrt{\varphi(\alpha)}
$$

Note that for functions $g_{\alpha}$ with $\left|1-\lambda g_{\alpha}(\lambda)\right| \leq 1$ relation (4.7) implies (6.4).

Theorem 6.2. Let $M_{\varphi, E}$ be given by (1.4) and let Assumption 1.1 be satisfied. Let $x_{\alpha}^{\delta}$ be the regularized approximation (6.2), let (3.3) hold and let $\alpha_{D}$ be the solution of equation (3.2). If assumption (6.4) is satisfied, then $x_{\alpha_{D}}^{\delta}$ is order optimal on the set $M_{\varphi, E}$ and

$$
\left\|x_{\alpha_{D}}^{\delta}-x^{\dagger}\right\| \leq c E \sqrt{\rho^{-1}\left(\frac{\delta^{2}}{E^{2}}\right)}
$$

with $c=\max \left\{C+1.6382, d_{\varphi}+0.6382\right\}$.

Proof. For method (6.2) assumption (4.4) holds true with $c_{0} \leq 0.6382$. Hence, for the case of $\alpha_{D} \geq \alpha_{0}$ with $\alpha_{0}$ given by (4.3) the proof of this theorem 
follows from Proposition 4.2. It remains to consider the second case $\alpha_{D} \leq \alpha_{0}$. From Proposition 6.1, the triangle inequality, estimation (4.6) and the identity

$$
\frac{\delta}{\sqrt{\alpha_{0}}}=E \sqrt{\rho^{-1}\left(\frac{\delta^{2}}{E^{2}}\right)}
$$

we obtain

$$
\begin{aligned}
\left\|x_{\alpha_{D}}^{\delta}-x^{\dagger}\right\| & \leq\left\|x_{\alpha_{0}}^{\delta}-x^{\dagger}\right\| \\
& \leq\left\|x_{\alpha_{0}}-x^{\dagger}\right\|+\left\|x_{\alpha_{0}}^{\delta}-x_{\alpha_{0}}\right\| \\
& \leq\left\|x_{\alpha_{0}}-x^{\dagger}\right\|+c_{0} E \sqrt{\rho^{-1}\left(\frac{\delta^{2}}{E^{2}}\right)}
\end{aligned}
$$

with $c_{0} \leq 0.6382$. Exploiting (6.2), (1.4), (6.4) as well as (4.3), we obtain in the second case

$$
\begin{aligned}
\left\|x_{\alpha_{0}}-x^{\dagger}\right\| & =\left\|\left[g_{\alpha_{0}}\left(A^{*} A\right) A^{*} A-I\right]\left[\varphi\left(A^{*} A\right)\right]^{1 / 2} v\right\| \\
& \leq d_{\varphi} E \sqrt{\varphi\left(\alpha_{0}\right)} \\
& =d_{\varphi} E \sqrt{\rho^{-1}\left(\frac{\delta^{2}}{E^{2}}\right)}
\end{aligned}
$$

Now (6.5) follows from $(6.6)-(6.7)$

Acknowledgement. M. Thamban Nair gratefully acknowledges the support received from German Academic Exchange Service (DAAD) to visit the Department of Mathematics, University of Kaiserslautern, during April-July 2002, under the Guest Professor's Programme (DAAD). In addition, the authors are grateful for the valuable hints of the anonymous referees that led to the improved version of the paper.

\section{References}

[1] Engl, H. W., Hanke, M. and A. Neubauer: Regularization of Inverse Problems. Dordrecht: Kluwer 1996.

[2] Groetsch, C. W.: The Theory of Tikhonov Regularization for Fredholm Equations of the First Kind. Boston: Pitman 1984.

[3] Hanke, M. and P. C. Hansen: Regularization methods for large-scale problems. Surv. Math. Ind. 3 (1993), $253-315$.

[4] Hofmann, B.: Regularization for Applied Inverse and Ill-Posed Problems (TeubnerTexte zur Mathematik: Vol. 85). Leipzig: B. G. Teubner Verlagsges. 1986.

[5] Hohage, T.: Regularization of exponentially ill-posed problems. Numer. Funct. Anal. \& Optimiz. 21 (2000), 439 - 464.

[6] Louis, A. K.: Inverse und schlecht gestellte Probleme. Stuttgart: Teubner 1989. 
[7] Mair, B. A.: Tikhonov regularization for finitely and infinitely smoothing operators. SIAM J. Math. Anal. 25 (1994), 135 - 147.

[8] Morozov, V. A.: On the solution of functional equations by the method of regularization. Soviet Math. Dokl. 7 (1966), $414-417$.

[9] Nair, M. T.: On Morozov's method for Tikhonov regularization as an optimal order yielding algorithm. J. Anal. Anw. 18 (1999), 37 - 46.

[10] Payne, L. E.: Improperly Posed Problems in Partial Differential Equations. Philadelphia: SIAM.

[11] Pereverzev, S. and E. Schock: Morozov's discrepancy principle for Tikhonov regularization of severely ill-posed problems. Numer. Funct. Anal. \& Optimiz. 21 (2000), $901-920$.

[12] Schock, E.: Approximate solution of ill-posed equations: arbitrary slow convergence vs. superconvergence. In: Constructive Methods for the Practical Treatment of Integral Equations (eds.: G. Hämmerlin and K. H. Hofmann). Basel: Birkhäuser Verlag, pp. $234-243$.

[13] Tautenhahn, U.: Optimality for ill-posed problems under general source conditions. Numer. Funct. Anal. \& Optimiz. 19 (1989), 377 - 398.

[14] Tikhonov, A. N. and V. Y. Arsenin: Solution of Ill-Posed Problems. New York: Wiley 1977.

[15] Vainikko, G. M. and A. V. Veretennikov: Iteration Procedures in Ill-Posed Problems (in Russian). Moscow: Nauka 1986.

Received 22.07.2002; in revised form 01.11.2002 